\title{
Properties and application of the unequal thickness two-component interference systems
}

\author{
I.V. Fekeshgazi, V.Yu. Pervak, Yu.A. Pervak \\ Institute of Semiconductor Physics of Ukrainian National Academy of Sciencies, \\ prosp. Nauki 45, Kiev, Ukraine, 01650. Phone 38044265 1817, Fax 380442658342. \\ E-mail:Pervak@dep48.semicond.kiev.ua
}

\begin{abstract}
The methods of synthesis and designing of the two-component unequal thickness multilayer interference systems as well as interconnection of their indices of layers are presented. A solution of the problem of suppressing high reflection zones at any harmonic frequency while maintaining high reflection at the operating frequency is proposed. The evolution of refractive zones at the inclined light incidence on the multilayer systems are studied. The results of the synthesis of antireflective coatings for some widely separated wavelengths are discussed. The application of results for the specific spectral characteristics and interference filters are proposed.
\end{abstract}

Keywords: multilayer interference coatings, multilayer mirror, prism interference polarization splitter.

Paper received 19.04.00; revised manuscript received 23.05.00; accepted for publication 30.06.00.

\section{Introduction}

The design of highly effective systems of the optical industry, nonlinear optics and laser techniques is closely connected with the development of optics for elements with different thin film coatings. Most frequently the periodic equal-thickness structures are used [1]. But those possess the limited spectral characteristics. In many cases the multilayer coatings with the definitely determined characteristics in several regions or with the broad bands of reflection or transmission are necessary. Sometimes these problems can be solved using the periodic multilayer structures where the individual layers have a different optical thicknesses [2]. And, what's more the seach of most optimal multilayer system is essentially simplified when the repetative periods have symmertic disposition of the layers [3-8]. In this paper, new achievements in modification of the spectral characteristics of multilayer dielectric systems that may be reached by changing the optical thicknesses of layers only within the thickness of repetative symmetric periods are presented.

\section{Theoretical background}

The designs of periodic two-component unequal thicknesses multilayer coatings can be represented as
$S\left\{[(1+\alpha) A(1-\alpha) B]^{m}\right\} S_{0}$ - for asymmetric systems and $S\left\{[(1+\alpha) A(1-\alpha) B]^{m}(1+\alpha) A\right\} S_{0}$ or $S\{(1+\alpha) A / 2[(1+\alpha) \times$ $\left.A / 2(1-\alpha) B(1+\alpha) A / 2]^{m}(1+\alpha) A / 2\right\} S_{0}$ - for symmetric ones, where $S$ is the substrate with refractive indices $n_{S}, S_{0}$ is the environment media with refractive indices $n_{0}$ (usually $S_{0}$ is air and $\left.n_{0}=1\right), m$ is the number of periods, $A$ and $B$ are the layers correspondently from the high or low refractive film forming matters with refractive indices $n_{A}$ and $n_{B}$ and quarter-wave optical thicknesses $n_{A} d_{A}=n_{B} d_{B}=\lambda_{0} / 4$ for definite wavelength $\lambda_{0}$ at $\alpha=0,-1<\alpha<1$ is the factor which indicate the interrelation between the thicknesses of layers [2-4].

The position and bandwidths of the high reflective bands of the unequal thickness asymmetric multilayer systems are determined from equation $[2,3]$

$\cos \left(\alpha \pi \lambda_{0} / \lambda\right)+T_{A B}=R_{A B} \sin \left(\pi \lambda_{0} / \lambda\right)$

where $T_{A B}$ and $R_{A B}$ are the energy transmission and reflection coefficients of the layer boundary, respectively. As can be seen the varying of $\alpha$ values the high reflective bands may be obtained at different integer values of $t=\lambda_{0} / \lambda$. Bandwidths of these regions depend on $\alpha$ and also on $n_{A}$ and $n_{B}$. The more is difference between the refractive indices, the wider is the reflection region.

To analyze the possibility of obtaining the transparency in a long wave region of spectrum, it is necessary to 


\section{I.V. Fekeshgazi et al.: Properties and application of the unequal thickness ...}

consider the spectral range of $\lambda_{0} / \lambda .<1$ in detail. It is known that the spectral characteristic of symmetric multilayer system is equivalent to single layer having a thickness of $m \lambda_{0} / 2$ similar to that of the stack and equivalent refractive index $N$ the same as for symmetric periods determined by $[1,4]$

$N=\eta_{A}[(a-b) /(a+b)]^{1 / 2}$,

where $a=\sin 2 \delta_{A} \cos \delta_{B}+(1 / 2)\left(\eta_{A} / \eta_{B}+\eta_{B} / \eta_{A}\right) \cos 2 \delta_{A} \sin d_{B}$;

$b=(1 / 2)\left(\eta_{A} / \eta_{B}+\eta_{B} / \eta_{A}\right) \sin \delta_{B}$

$\delta_{A}=\left(2 \pi n_{A} d_{A} \cos \theta_{A}\right) / \lambda ; \quad \delta_{B}=\left(2 \pi n_{B} d_{B} \cos \theta_{B}\right) / \lambda ;$

$\eta_{A, B}=n_{A, B} \cos \theta_{A, B}$ for $s$-polarization and

$\eta_{A, B}=n_{A, B} / \cos \theta_{A, B}$ for $p$-polarization,

$\eta_{A}$ and $\eta_{B}$ - effective refractive indecies, $\delta_{A}$ and $\delta_{B}$ phase thicknesses, $d_{A}$ and $d_{B}-$ geometric thicknesses, $\theta_{A}$ and $\theta_{B}$-angles which definite the direction of the light propagation in the layers of $A$ and $B$, respectively.

It's easily seen that any required value of $N$ can be achieved under the suitable choice of parameter $a$ and at fixed $n_{A}$ and $n_{B}$. Moreover, $N$ can be obtained both with negative and positive dispersion in some limited spectral range depending on $n_{A}$ and $n_{B}$ ratio.

In a general case, the reflection and transmission spectra of any periodic multilayer system consist of repeated periodic alternated zones of the high reflection and transmission. Position of short and long wavelength zone boundaries as well as of their bandwidths can be determined from analysis of the argument of the Chebychev polynomes $[1,2]$. The spectral location of these boundaries can be find from equation [4]

$\cos \beta_{A} \cos \beta_{B}-0.5\left(\eta_{A} / \eta_{B}+\eta_{B} / \eta_{A}\right) \sin \beta_{A} \sin \beta_{B}= \pm 1$

where $\beta_{A}=0.5 \pi\left(\lambda_{0} / \lambda\right)(1+\alpha) \cos \theta_{A}, \beta_{B}=0.5 \pi\left(\lambda_{0} / \lambda\right)(1-\alpha) \cos \theta_{B}$.

In some special cases, the analytical expression for bandwidth of high reflection zone can be obtained. For example, such expressions are well known for the quarter waves stack at normal incidence and also for unequal thickness periodic structure of form $S(A B)^{m}$ [2] . But for arbitrary values of incidence angle $\theta_{0}$ and parameter $\alpha$ the exact position of boundaries and bandwidth of the reflection zone are obtained by numerical solution of expression (3).

\section{Suppressing of the high reflective zones}

The both structures (asymmetric and symmetric) at $\alpha=0$ represent periodic quarter-wave stacks of the form $S\left\{(A B)^{m}\right\} S_{0}$ and $S\left\{(A / 2)[(A / 2) B(A / 2)]^{m} A / 2\right\} S_{0}$. In fact the symmetric stacks differ from the asymmetric ones by the presence of the additional A layer. Because of $n_{A} d_{A}=$ $n_{B} d_{B}=\lambda_{0} / 4$ the both systems are characterized by the spectral dependencies of reflection $R\left(\lambda_{0} / \lambda\right)$ that is sym- metric in relation to the maxima localized near the odd integer values of $t=\lambda_{0} / \lambda$ or the transmission $T\left(\lambda_{0} / \lambda\right)$ ones localized at even value of $t$ (Fig. 1a). Ordinarilly, the multilayer coatings with broad bands of transmission or reflection in different regions of spectrum are necessary for many practical applications. To obtain a transparency at given integer $t$ and the shorter wavelength $\lambda$ than $\lambda_{0}$, it is necessary to suppress the corresponding zone of high reflection. For the even $t$ the depression takes place at $\alpha=2 k / t[k=0,1, \ldots, t / 2]$ and for odd ones - at $\alpha=$ $(2 k+1) / t[k=0,1, \ldots, t / 2](2 k+1) / t[k=0,1, . .,(t-1) / 2]$. At the same time while depressing high reflection zones, there is some decrease of the reflectivity also at $t=1$. The more $a$, the more strong decreasing is obtained (Fig. 1d and $\mathrm{f}$ ). Beside that the form of spectral dependencies differ for the case $n_{A} d_{A}>n_{B} d_{B}$ and $n_{A} d_{A}<n_{B} d_{B}$. This feature is illustrated in Figs $1 \mathrm{~b}$ and $\mathrm{c}$ for two assemblies with the zones depression at $t=3$. Their structure being of the $S(1.33 A 0.67 B)^{m} 1.33 A$ (Fig. $\left.1 b\right)$ and $S(0.67 A 1,33 B)^{m} 0,67 A$ (Fig. 1c) at $a=0,33$ and $S(1.2 A 0.8 B)^{m} 1.2 A$ (Fig. 1d) and $S(0.4 A 1.6 B)^{m} 0.4 A$ (Fig. 1f) at $\alpha=0.2$ and $\alpha=0.6$ correspondingly. As can be seen the high reflective band at $t=3$ is depressed at $\alpha=0,33$ and the high reflectance band at $t=5$ is depressed at $\alpha=0.2$ and $\alpha=0.6$. For the second case the reflectivity at $t=1$ is greater at $\alpha=0.2$ than at $\alpha=0.6$.

It is important that the spectral characteristics of the same multilayer coatings are different for case of equal

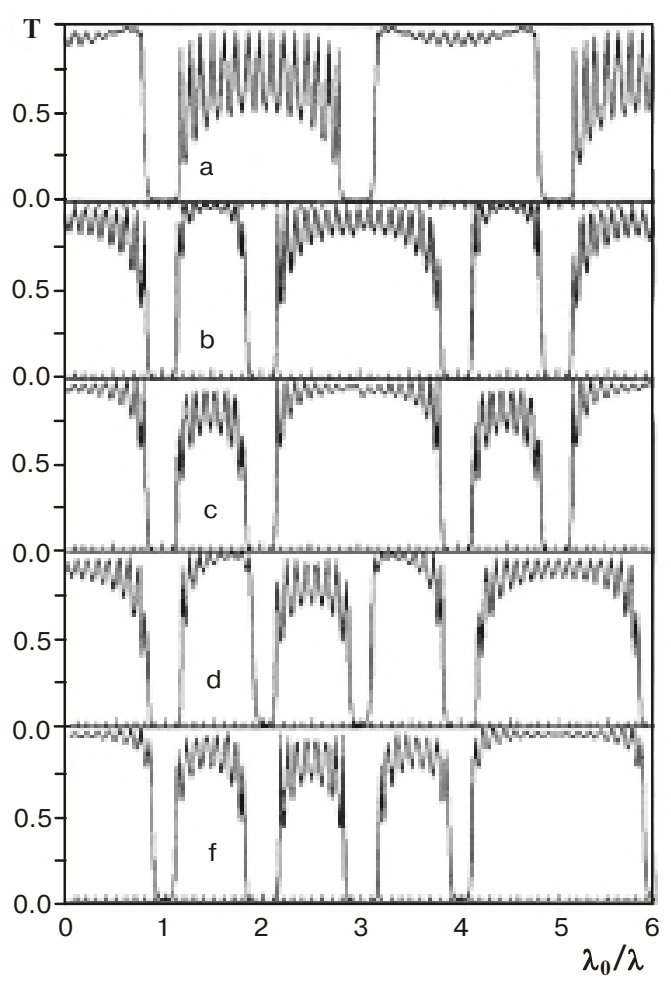

Fig. 1. Transmittence of the some design at $n_{S}=1.52, n_{A}=2.3, n_{B}$ $=1.38, m=8$ and $a=0$ (a), $a=0.33$ (b), $a=-0.33$ (c), $a=0.2$ (d), $a=0.6$ (f). 


\section{I.V. Fekeshgazi et al.: Properties and application of the unequal thickness ...}

refractive indeces $n_{S}=n_{S o}$ and for case of unequal refractive indices $n_{S} \neq n_{S \mathrm{o}}$.

For the case of equal refractive indices $n_{S}=n_{S \mathrm{o}}$ the dependencies of the position of the first high reflection zone boundaries from parameter $a$ is shown in Fig. 2, and the spectral dependencies of the reflection coefficient for multilayer structures $S(A B A)^{m} S_{0}$ at different incidence angles $\theta_{0}$ are shown in Fig. 3. The solid curves in Fig. 2 correspond to the first high reflection zone boundaries for $s$ polarization of light wave and dotted curves for $p$ polarization. It is seen that position and bandwidth of high reflection zones essentially depend on angle $\theta_{0}$ and value of parameter $\alpha$. The high reflection zones are shifted towards the short wavelengths with increasing $\theta_{0}$.

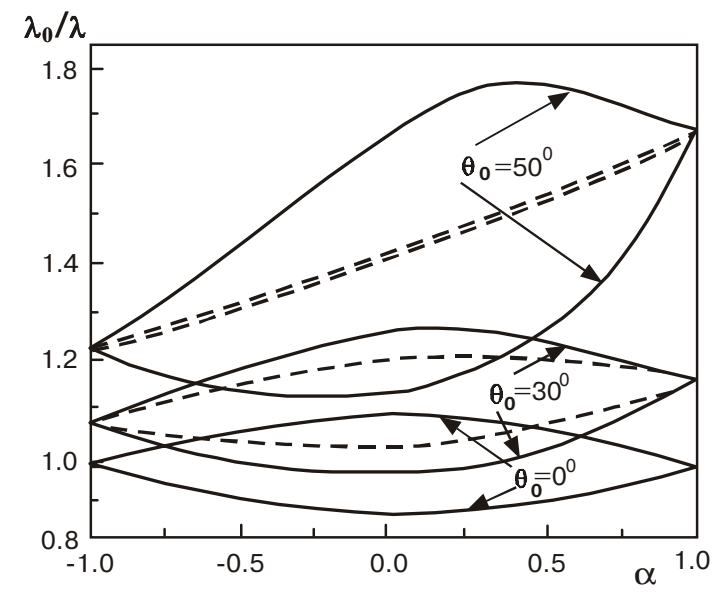

Fig. 2. Boundaries position of the first high reflection zone for different incidence angles $\theta_{0}$.

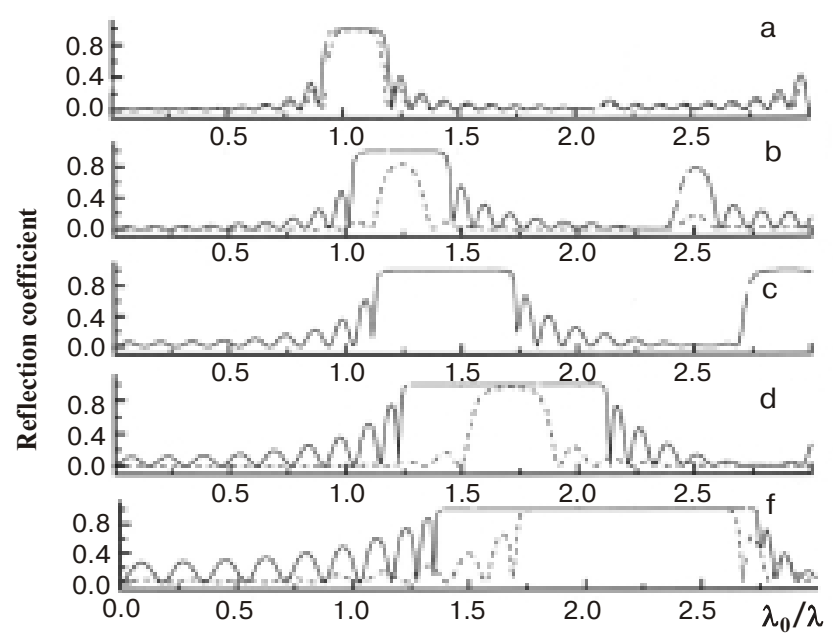

Fig. 3. The spectral dependences of the reflectance of the multilayer structures $\mathrm{S}(\mathrm{ABA}){ }^{10} \mathrm{~S}$ for different incidence angles $\theta_{0}: 20^{\circ}$ (a), $40^{\circ}$ (b), $50.6^{\circ}$ (c), $60^{\circ}$ (d), $70^{\circ}$ (f). $n_{S}=1.52, n_{A}=1.45, n_{B}=2.0$. Solid curves - $s$ polarization, dashed curves - $p$ polarization.
Under increasing $\theta_{0}$ from 0 to the Brewster angle $\theta_{B r}$ (for example, $\theta_{B r}=50.6^{\circ}$ for $n_{s}=n_{S o}=1.52, n_{A}=1.45$, $\left.n_{B}=2.0\right)$, the reflective zone bandwidth $\mathrm{D}\left(\lambda_{0} / \lambda\right)$ is increased for $s$-polarization and decreased to zero for $p$ polarization ( $p$ component is full transmitted). At further increasing $\theta_{0}$ the high reflection zone bandwidths for both polarization are increased.

For the case of unequal refractive indices $n_{S} \neq n_{S \mathrm{o}}$ the decrease of the reflective zone bandwidth $\mathrm{D}\left(\lambda_{0} / \lambda\right)$ for $p$ polarization is more slow then it is for $n_{S}=n_{S \mathrm{o}}$ at the same optical parameters of the multilayer system. Such multilayer systems $\left(n_{S} \neq n_{S o}, n_{S o}=1, \mathrm{~S}_{0}-\right.$ air $)$ are more effective for designing reflective filters.

\section{Anti-reflective coatings for several spectral regions}

The possibility of obtaining $N$ with any required values enable to use such symmetrical multilayer system as antireflective coatings simultaneously on several wavelengths. It was obtained that the possible values of $N$ can not be less of smaller and more of greater $n_{A}$ or $n_{B}$ at $\lambda_{0} / \lambda \rightarrow 0$. At $\alpha=0$ the $N=\left(n_{A} n_{B}\right)^{1 / 2}$ and if $n_{A}>n_{B}$ and $\alpha$ is changed from -1 to 1 then $N$ value increases from $n_{A}$ to $n_{B}$ for period $(1+\alpha) B / 2(1-\alpha) A(1+\alpha) B / 2$ and decreases from $n_{B}$ to $n_{A}$ for period $(1+\alpha) A / 2(1-\alpha) B(1+\alpha) A / 2$. The spectral dependence of the reflectance $R$ of the multilayer structure $S\left\{(1+\alpha) A / 2[(1+\alpha) A / 2(1-\alpha) B(1+\alpha) A / 2]^{m} \times\right.$ $\times(1+\alpha) A / 2\}$ that consists of $m$ symmetrical period $(1+\alpha) A / 2(1-\alpha) B(1+\alpha) A / 2$ on substrate $S$, is the oscillating function between two envelopes $R_{1}$ and $R_{2}$ (Fig. 4). The envelope $R_{1}$ is the spectral dependence of substrate reflectance without multilayer structure, and $R_{2}$ is the spectral dependence of substrate reflectance with quarter-wavelength film with reflective index $N$. If the struc-

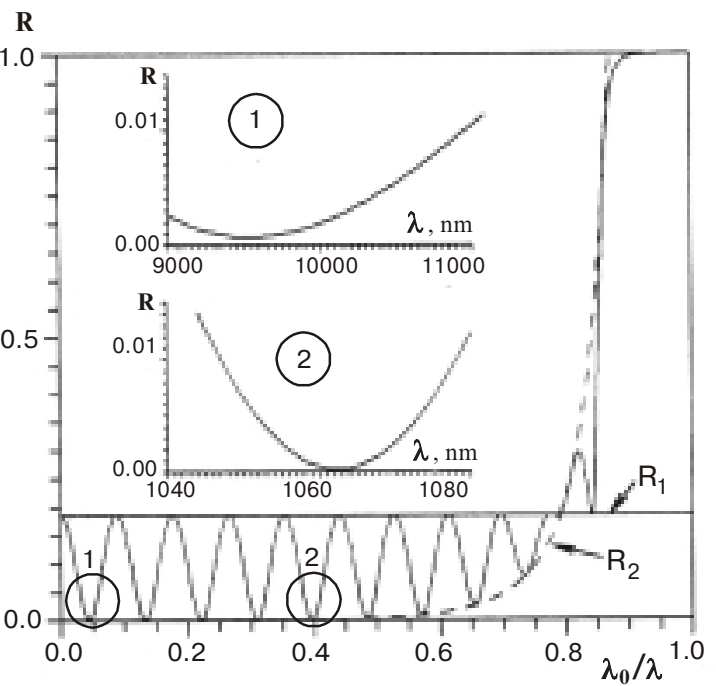

Fig. 4. The spectral dependences of reflactance $(R)$ of the multilayer system $\mathrm{S}(\mathrm{ABA})^{11}$, substrate $\left(R_{1}\right)$ and envelope $\left(R_{2}\right)$. 
ture parameters are chosen in such way that the substrate refractive index $n_{S}>N$, then $R$-function minima would be on $R_{2}$ and $R$-function maxima would be on $R_{1}$. The values of $R=0$ is obtained at $N=\left(n_{S}\right)^{1 / 2}$ in the points of minima. Their quantity is determined by the full optical thickness of the multilayer structure $[(1+\alpha) A / 2(1-\alpha) B(1+\alpha) A / 2]^{m}$. As the optical thickness of $(1+\alpha) A / 2(1-\alpha) B(1+\alpha) A / 2$ period is $\lambda_{0} / 2$, then in practice the repeated period multiplicity $m$ determined the extrema number obtain the minima $R$ at assigned wavelengths $\lambda_{i}\left(\lambda_{i}>\lambda_{0}\right)$ it's necessary that they will be described by the expression $\lambda_{i}=2 m \lambda_{0} /(2 r-1)$, where $r=1,2,3, \ldots$

Using this method the special anti-reflective coating was received. It is anti reflecting for two wavelenghts $\lambda_{1}=10.6$ and $\lambda_{2}=1.06 \mu$.

Its structure is $S\{(1+\alpha) A / 2(1-\alpha) B(1+\alpha) A / 2\}^{11}$, and parameters are as follows: $n_{A}=1.35, n_{B}=2.3, n_{S}=2.52$, $\alpha=0.496, \lambda_{0}=420.8 \mathrm{~nm}$.

Respective reflections are $R(\lambda=1.06 \mathrm{~m})=0.0003$ and $R(\lambda=10.6 \mu)=0.006$ (Fig. 4$)$.

Thus, by the variation of $\alpha, \lambda_{0}$ and $\mu$ factors the coatings with broad transparency bands or with simultaneous antireflection at some wavelengths can be realized.

\section{Optimization of the design of prism interference polarization splitters}

The problems of production of linearly polarized light and separation of light fluxes with respect to polarization using of interference multilayer coatings are extensively studied [6-20]. In the prism and plate-type designs of interference polarizers (IPs), which are most widely used, the radiation incident on a multilayer coating is separated into the reflected and transmitted beams polarized in mutually orthogonal directions. The coating of an IP represents a multilayer two-component interference system formed by alternating layers of materials with high and low refractive indices $n_{H}$ and $n_{L}$. The prism interference polarizers are used for the production of linearly polarized light in devices working at fixed wavelengths and in a wide spectral region. A conventional design of such an IP represents two rectangular prisms glued together, with a multilayer coating deposited onto the hypotenuse faces of one prism or both of them. To increase transmitted light intensity, some IPs have an optical contact between the prisms and not a glued pair of prisms [17] or use a special cell filled with a liquid, thin substrate as well as multilayer coating placed inside the liquid and oriented at a certain angle to the incident radiation, instead of the prisms $[16,19]$. The optical thicknesses of the layers are determined by the spectral region of the maximum values of the degree of polarization. Their values in the direction of light flux propagation are chosen equal to

$n_{H} d_{H}=n_{L} d_{L}=0.25 \lambda_{0}$,

where $d_{H}$ and $d_{L}$ are the geometrical thicknesses of the layers $H$ and $L$, respectively, and $\lambda_{0}$ is the wavelength corresponding to the center of the spectral region with the maximum degree of polarization.

A high degree of polarization of reflected and transmitted radiation is provided in the case where the reflection of radiation from the interfaces of the structure takes place at the Brewster angles

$\varphi_{H}=\arctan \frac{n_{L}}{n_{H}}, \varphi_{L}=\arctan \frac{n_{H}}{n_{L}}$,

Here, $\varphi_{H}$ and $\varphi_{L}$ are the angles determining the directions of propagation of radiation in the layers $H$ and $L$, respectively. It is more common to use another representation of condition (5), namely,

$\frac{n_{H}}{\cos \varphi_{H}}=\frac{n_{L}}{\cos \varphi_{L}}$.

Conditions (5) and (6) can be fulfilled by way of matching the refractive indices of the prisms $\left(n_{S}\right)$ and the layers $\left(n_{H}\right.$ and $\left.n_{L}\right)$ and, moreover, the angle of incidence on a multilayer coating $\varphi_{S}$, which is commonly chosen equal to the prism angle $\varphi_{p}$. This is possible in the case where one satisfies the equality [5]

$$
n_{S}^{2} \sin ^{2} \varphi_{S}=\frac{n_{H}^{2} n_{L}^{2}}{n_{H}^{2}+n_{L}^{2}}
$$

There are three main types of prism IPs, which differ by the conditions of transmission and reflection of light at the layer-layer and prism-coating interfaces. They are characterized by different spectral dependences of the degrees of polarization for reflected $\left(P_{R}\right)$ and transmitted $\left(P_{T}\right)$ light, which are determined by the expressions

$$
P_{R}=\frac{R_{s}-R_{p}}{R_{s}+R_{p}} \quad \text { and } \quad P_{T}=\frac{T_{s}-T_{p}}{T_{s}+T_{p}}
$$

where $R_{s}$ and $R_{p}$ are the reflectivities and $T_{s}$ and $T_{p}$ are the transmittances for the $s$ and $p$ components, respectively.

The $A$ type IP (IPA) corresponds to the case where conditions (5) and (6) for the Brewster angles are fulfilled at all the interfaces (the prism-coating and $H$ layer $-L$ layer interfaces). In this case, $n_{S}=n_{L}$ and the IPA provides the maximum degree of polarization in the reflected and transmitted beams $\left(P_{R}=P_{T}\right)$, with the angle between these beams $\varphi_{R T} \neq 90^{\circ}$ because $\varphi_{p}=\varphi_{S} \neq 45^{\circ}$ (if $\varphi_{p}=\varphi_{S}=$ $45^{\circ}$, we find, in view of (6) and (7), that $n_{S}=n_{L}=n_{H}$ ).

The $B$ type IP (IPB) corresponds to the case where the conditions for the Brewster angles are fulfilled at the layerlayer interfaces only. In this case, $n_{S} \neq n_{L} \neq n_{H}$. The IPB provides the maximum degree of polarization for the transmitted beam only, and the degree of polarization for the reflected beam is $P_{R}<P_{T}$. However, one can obtain in this case the angle beams $\varphi_{R T}=90^{\circ}$, provided $\left(\varphi_{p}=\varphi_{S}=45^{\circ}\right.$ (polarization cubes).

The $C$ type IP (IPC) corresponds to the case where the conditions for the Brewster angles are fulfilled on none of the interfaces. 


\section{I.V. Fekeshgazi et al.: Properties and application of the unequal thickness ...}

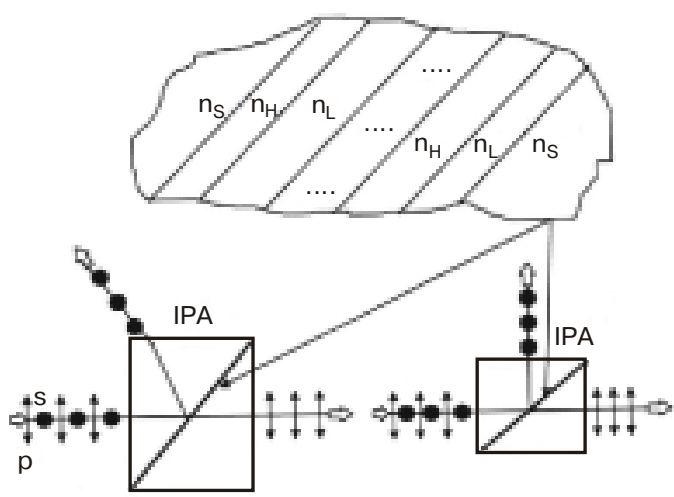

Fig. 5. Design of prism interference polarizers.

The schematic designs of IPA and IPB and their principles of operation are illustrated in Fig. 5. The design of an IPA may be similar to the designs of IPA and IPB, but in this case it provides efficient operation at fixed wavelengths or in a very narrow spectral region only. The spectral characteristics of IPA, IPB, and IPC are substantially different (Fig. 6). These distinctions are associated mainly with a strong dependence of the transmittance on the angle of incidence for the $p$ component of a light beam. In real conditions, the maximum width of the operating region of IPA and IPB, in which the highest intensities and degrees of polarization for the transmitted and reflected beams are obtained, is limited by the width of the region providing a high reflectivity for the $s$ component of light.

However, the latter region can be expanded by deposition of several multilayer systems with quarter-wavelength layers for several fixed wavelengths. In this case, the transmittance for the $p$ component is independent of thickness and number of layers because of the fulfillment of the conditions for the Brewster angles at the layerlayer interfaces. In the case of the IPC, the width of the operating region of a polarizer increases with decreasing the difference between the angles of incidence and the Brewster angles. Sometimes, the operating region of an IPC is widened owing to the use of multilayer coating with unequal thicknesses parameters of which are determined by various optimization methods [20]. The main disadvantage of such IPCs is that their fabrication requires a considerable complication of technology, which is associated with the necessity of excluding the smallest deviations in the thickness of layers being deposited.

Up to the present time, the problem of designing interference polarizers providing the maximum possible degrees of polarization (100\%) for orthogonally propagating transmitted and reflected beams was unsolved. At the same time, the use of polarization splitters, which split the incident beam into two beams with orthogonal
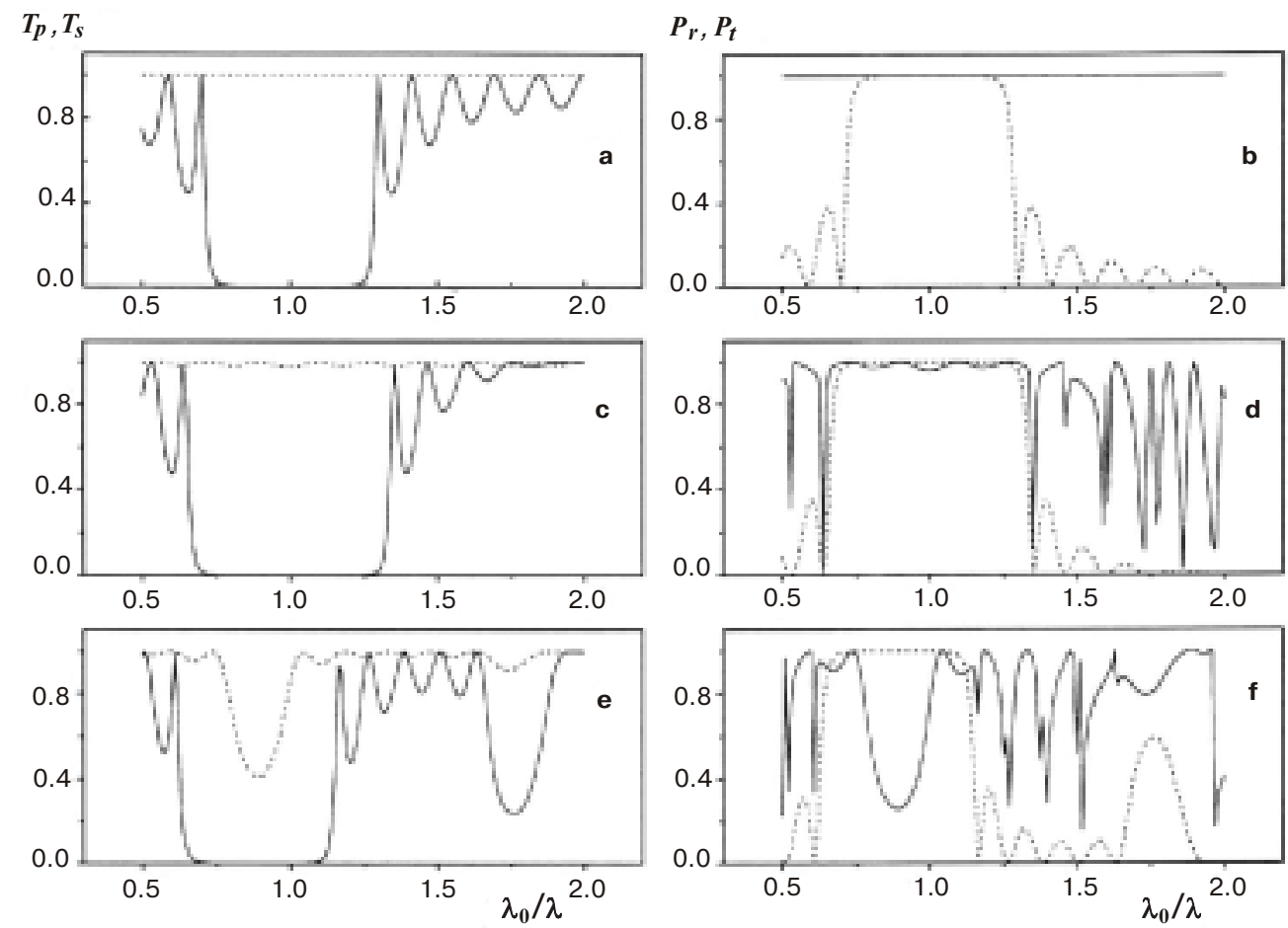

Fig. 6. The spectral dependences of (a, c, e) the transmittances $T_{s}$ and $T_{p}$ for the $s$ (solid curves) and $p$ (dashed curves) components of radiation, respectively, and (b, d, f) the degrees of polarization $P_{T}$ and $P_{R}$ for the transmitted (solid curves) and reflected (dashed curves) beams, respectively, for $(\mathrm{a}, \mathrm{b})$ IPA, $(\mathrm{c}, \mathrm{d}) \mathrm{IPB}$, and $(\mathrm{e}, \mathrm{f})$ IPC with 15 -layer coatings $(\mathrm{HL})^{7} \mathrm{H}$. The reflection from cathetus surfaces is ignored. For all Ips, $n_{S}=1.673, n_{H}=2.3, n_{L}=1.38 ; \varphi_{p}=\varphi_{S}=51.125^{\circ}(\mathrm{IPA}), 45^{\circ}$ (IPB), $60^{\circ}$ (IPC). 


\section{I.V. Fekeshgazi et al.: Properties and application of the unequal thickness ...}

polarizations and directions of propagation, makes it possible to considerably simplify the design of laser interferometers [14]. However, the $B$ type interference polarizers, which are suitable for this purpose, have a substantial deficiency. Their spectral dependences of $T_{p}$, transmittance for the $p$ component, and $P_{R}$, the degree of polarization of a reflected beam, are oscillating functions, which is caused by the fact that it is impossible to fulfil the condition for the Brewster angle (5) at the prismcoating interfaces.

Indeed, for each prism-coating interface of the IPB with the angles $\varphi_{p}=\varphi_{S}=45^{\circ}$, the maxima of the function $T_{p}\left(\lambda_{0} / \lambda\right)$ are found on the straight line $T_{p}=1$, and the minima are found on the straight line

$$
T_{p}=\frac{4 \eta_{S}^{2} \eta_{H}^{2}}{\left(\eta_{S}^{2}+\eta_{H}^{2}\right)^{2}}=\frac{4 \eta_{S}^{2} \eta_{L}^{2}}{\left(\eta_{S}^{2}+\eta_{L}^{2}\right)^{2}}
$$

Here, $\eta_{S}=n_{S} \cos \varphi_{S}, \eta_{H}=n_{H} \cos \varphi_{H}$ and $\eta_{L}=n_{L} \cos \varphi_{L}$ are the effective refractive indices for the $p$ component of radiation in the prism, the layer $H$, and the layer $L$, respectively. Using the law of sines

$n_{S} \sin \varphi_{S}=n_{H} \sin \varphi_{H}=n_{L} \sin \varphi_{L}$

and relation (7), one can represent expression (9) in the form

$$
T_{p}=\frac{16 x^{2}\left(x^{2}+1\right)^{2}}{\left[4 x^{2}+\left(x^{2}+1\right)^{2}\right]^{2}}
$$

where $x=n_{H} / n_{L}$. From the analysis of expression (11) it follows that the greater is the ratio $n_{H} / n_{L}$, the lower is the transmittance $T_{p}$. If the amplitude of oscillations of $T_{p}$ in the IPB is to be not greater than $1 \%$, the ratio $n_{H} / n_{L}$ must be smaller than 1.45. For zinc sulfide $\mathrm{ZnS}\left(n_{H}=2.3\right)$ and magnesium fluoride $\mathrm{MgF}_{2}\left(n_{L}=1.38\right)$ as an example, which are commonly used as film-forming materials, $n_{H} / n_{L}=1.667$, and the corresponding value of $T_{p, \min }$ is 0.984 . At the same time, a decrease of the ratio of refractive indices of layers causes narrowing the operating region of the IPB $\Delta\left(\lambda_{0} / \lambda\right)$.

Moreover, a decrease $n_{H} / n_{L}$ results in the fact that the achievement of rather high values of reflectivity for the $s$ component as well as degrees of polarization for reflected and transmitted radiation requires an increase of the number of layers in an interference coating. The latter is able to cause considerable technological problems in the fabrication of a polarizer, which are associated with its strength and reliability.

From (9) it follows that the equality of effective refractive indices $n_{S}=n_{H}$ gives the transmittance $T_{p}=1$, but, as shown above, this cannot be realized in the IPBs for the angles $\varphi_{p}=\varphi_{S}=45^{\circ}$. Because of this it seems reasonable to use the methods known in the theory of antireflection coatings in order to suppress oscillations of $T p$ by matching a prism to a coating. As will be shown below, in the majority of cases it suffices to introduce one matching layer refractive index of which for the $p$ component is determined from the condition specifying the relation between the effective indices of the matching layer, prism and layer with a high refractive index

$\eta_{C}=\left(\eta_{S} \eta_{H}\right)^{1 / 2}$

Here, $\eta_{C}=n_{C} / \cos \varphi_{C}, n_{C}$ is the refractive index of the matching layer, and $\varphi_{C}$ is the angle specifying the direction of propagation in this layer. The $n_{C}$ can be obtained from the expression for the refractive index of the matching layer (12):

$n_{C}=\left\{n_{H} n_{L}\left[1 \pm \frac{n_{H}-n_{L}}{\left(n_{H}^{2}+n_{L}^{2}\right)^{1 / 2}}\right]\right\}^{1 / 2}$,

which shows the existence of two values of this refractive index $n_{C}$. The optical thickness of the matching layer is chosen so that its value in the direction of propagation of light be equal to a quarter of the wavelength $\lambda_{0}$ :

$n_{C} d_{C} \cos \varphi_{C}=0.25 \lambda_{0}$.

Fig. 7 presents the spectral dependences of transmittances and degrees of polarization calculated for IPBs with conventional structures of the multilayer coating and structures having an additional matching layer. It should be noted that the spectral dependences of transmittance $T_{p}$ for both values $n_{C 1}$ and $n_{C 2}$, which are the solutions of (13), are totally identical, whereas the spectral dependences of $T_{s}$ (the transmittance for the $s$ component), have a small mutual shift. In the operating spectral region of the polarizer, the corresponding dependences of $T_{s}$ are shifted in the direction of small $\lambda_{0} / \lambda$ (long-wavelength region) for $n_{C 1}\left(n_{C 1}<n_{C 2}\right)$ and in the direction of large $\lambda_{0} / \lambda$ (short-wavelength region) for $n_{C 1}$. This is associated with the fact that the effective refractive index for the $s$ component that corresponds to $n_{C 1}$ is smaller that for $n_{C 2}$. The possibility of choosing the refractive index for the matching layer simplifies the procedure of determination of optimum parameters of polarization splitters.

\section{Prism polarizers for broad aperture beams.}

The optical properties of IPs depend on the aperture of an incident beam because its increase causes changes in angles of incidence on a coating, effective optical thicknesses of layers, and reflectivities for polarization components. As the angle of incidence $\varphi_{S}$ decreases, the operating spectral region of an IP $\Delta \lambda$, becomes narrower. This is accompanied by an increase of $T_{s}$ and a decrease of $T_{p}$ as well as the degree of polarization of transmitted radiation $P_{T}$. As the angle of incidence $\varphi_{S}$ increases, the width $\Delta \lambda$ and the degree of polarization increase, whereas $T_{s}$ and $T_{p}$ decrease. In the case where one uses a divergent beam, the IPA is characterized by lower losses for the $p$ components than the values observed for the IPB 


\section{I.V. Fekeshgazi et al.: Properties and application of the unequal thickness ...}
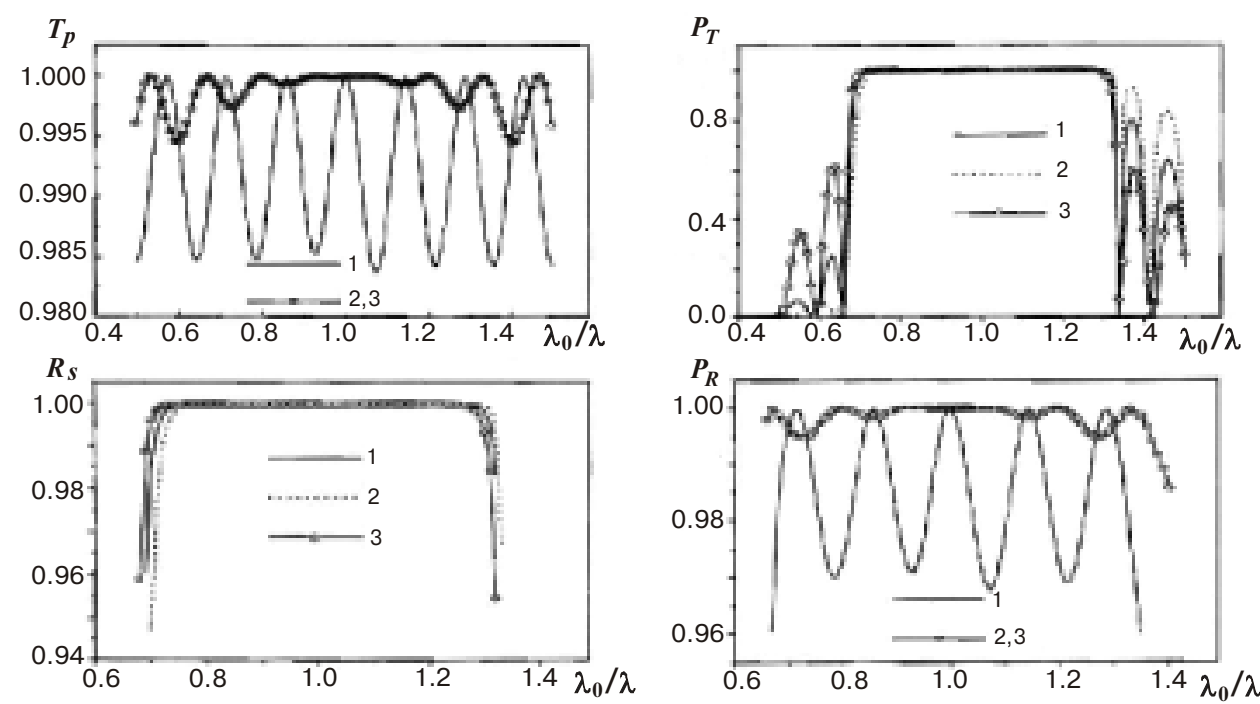

Fig. 7. The spectral dependences of the transmittances $T_{p}$ and reflectances $R_{s}$ for the $p$ and $s$ polarized light, respectively, and the degrees of polarization $P_{T}$ and $P_{R}$ for the transmitted and reflected beams, respectively, for Ips with multilayer coatings: (1) (0.5LH0.5L) ${ }^{7}$, (2) $\mathrm{C}_{1}(0.5 \mathrm{LH} 0.5 \mathrm{~L})^{7} \mathrm{C}_{1}$ and (3) $\mathrm{C}_{2}(0.5 \mathrm{LH} 0.5 \mathrm{~L})^{7} \mathrm{C}_{2} . n_{S}=1.673, n_{H}=2.3, n_{L}=1.38 ; n_{C 1}=1.444, n_{C 2}=2.064 ; n_{H} d_{H}=1.666\left(\lambda_{0} / 4\right)$; $n_{L} d_{L}=1.942\left(\lambda_{0} / 4\right) ; n_{C 1} d_{C 1}=1.744\left(\lambda_{0} / 4\right) ; n_{C 2} d_{C 2}=1.22\left(\lambda_{0} / 4\right)$.

[13]. For the deviations of the angle of incidence from the optimum value by 1 to $2^{\circ}$, the spectral dependences of $P_{R}$ and $T_{p}$ have dips, which reach $10-20 \%$. The plate-type polarizers are characterized by a considerable achievement. Their angular aperture for incident light was increased up to $5-10^{\circ}$ with retention of high $T_{p}>95 \%$ [12]. However, in the case of interference coatings formed by layers with unequal thicknesses and symmetric periods, the aperture of incident radiation can be increased up to 20-24 ${ }^{\circ}$ with preservation of spectral characteristics of a polarizer in the operating spectral region $[6,7]$. It follows from (2) and (5) that for $p$ component of radiation, the equivalent refraction index $N_{p}=\eta_{A}=\eta_{B}$ and does not depend on a thickness of layers when realizing conditions (5). If the prism refraction index $n_{S}$ is selected in such a manner that it satisfies the condition of Brewster angles on the boundary the prism - coating, then transmittance of the $p$ component radiation $T_{p}=1$. In other cases transmittance will be oscillating function with maxima on straigt line $T_{p}=1$ and minima on line

$T_{p}^{\min }=4 \eta_{S}^{2} N_{p}^{2}\left(\eta_{S}^{2}+N_{p}^{2}\right)^{2}$

where $\eta_{S}=n_{S} / \cos \varphi_{S}-$ effective prism refraction index.

Thus, when conditions (5) realized an equivalent refraction index of a multilayer system and transmittance of $p$ component do not depend neither on the number of layers, nor their thickness. Their values are selected from condition of deriving of a maximum reflection for $s$ component radiations. Therefore, operating spectral region of IP is a band of a high reflection of multilayer coating for $s$ component radiation.
At angles of incidence, when the conditions of Brewster angles are not fulfilled, the equivalent refraction index essentially depends on a thickness of layers. However, also in this case, practically for any pair of filmforming substances it is possible to reach an obvious equality $\eta_{S}=N_{p}$ by changing relation of optical thicknesses of separate layers in the period at preservation of a period optical thickness. However, spectral regionin which it is possible to receive high degrees of polarization for transmitted and reflected light is already limited by the long wavelength boundary of zones of a high reflection of $p$ and $s$ component at the angle of incidence on the coating $\varphi>\varphi_{p}$ (where $\varphi_{p}$ - angle of a prism selected so that a condition (5) to be fulfilled ), and at $\varphi<\varphi_{p}$ - the appropriate short-wave boundaries.

In Fig. 8 shown are spectral dependences of the transmittances for the $s$ and $p$ component of radiation at various angles of incidence on multilayer coating. Prisms of IPs were made from an optical glass K-8 $\left(n_{S}=1.52\right)$ with an angle $\varphi_{p}=51.125^{\circ}$. The angle $\varphi_{p}$ is selected so that the conditions of Brewster's angles (5) in multilayer coating were fulfilled. Multilayer interference coating consists of alternated layers of a magnesium fluoride $\mathrm{MgF}$ $\left(n_{A}=1.38\right)$ and zinc sulphide $\operatorname{ZnS}\left(n_{B}=2.3\right)$ and has a structure $(\mathrm{ABA})^{7}$ (optical thicknesses are $n_{A} d_{A}=1.25 \lambda_{0} / 4$ and $\left.n_{B} d_{B}=0.723 \lambda_{0} / 4\right)$. It is visible, that at $\varphi=\varphi_{p}$ transmittance $T_{p}$ is poorly oscillating function with $T_{p}^{\min }=$ 0.9897 . With increasing $\varphi$ the long wavelength boundaries of a high reflection zones are displaced in a shortwave side (to large values of $\lambda_{0} / \lambda$ ). Thus, when of an angle $\varphi$ is changed from $51.125^{\circ}$ up to $65^{\circ}$, spectral range in which $T_{p}>$ and $T_{s}<10^{-4}$ is decreased almost twice. It is in 
I.V. Fekeshgazi et al.: Properties and application of the unequal thickness ...

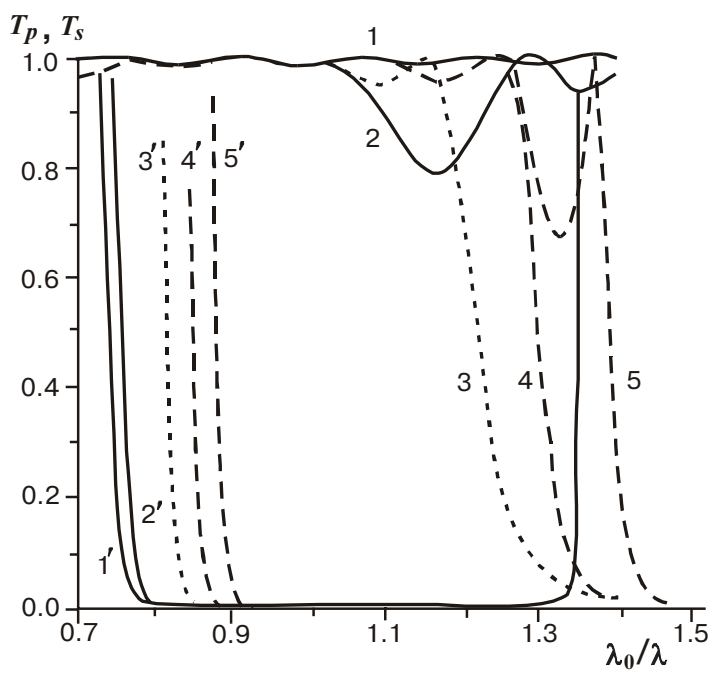

Fig. 8. The spectral dependences of the transmittances $T_{p}(1-5)$ and $T_{S}\left(1^{\mathrm{a}}-5^{\mathrm{a}}\right)$ for the $p$ and $s$ polarized light, respectively, for Ips with multilayer coatings $(\mathrm{ABA})^{7}$ at incidence angles: $51.125^{\circ}$ $\left(1,1^{\mathrm{a}}\right), 53^{\circ}\left(2,2^{\mathrm{a}}\right), 59^{\circ}\left(3,3^{\mathrm{a}}\right), 62^{\circ}\left(4,4^{\mathrm{a}}\right), 65^{\circ}\left(5,5^{\mathrm{a}}\right) . n_{B}=2.3$, $n_{A}=1.38 ; n_{A} d_{A}=1.25\left(\lambda_{0} / 4\right) ; n_{B} d_{B}=0.723\left(\lambda_{0} / 4\right)$.

limits of $(0.9-1.05) \lambda_{0} / \lambda$ and $(0.78-1.32) \lambda_{0} / \lambda$ at $\varphi=65^{\circ}$ and $\varphi=51.125^{\circ}$, accordingly. The increasing $\varphi$ within stated limits corresponds to increasing of an angle of incidence on a IP's leg surface from $0^{\circ}$ up to $21.38^{\circ}$ and increasing of the prism effective refraction index $\eta_{S p}$ for $p$ component from 2.422 up to 3.597. Optical thicknesses of interference coating were selected so that an equivalent refraction index $N_{p}\left(\lambda_{0} / \lambda=1\right) \approx \eta_{S p}$ at $\varphi=65^{\circ}$. At such choice in all considered range of angles $\varphi$, the equivalent refraction index $N_{p}$ is close to an appropriate value $\eta_{S p}$. The values $\eta_{S p}$ at $\varphi=51,53,59,62$ and $65^{\circ}$ are equal $2.422,2.523,2.951,3.238$ and 3.597 respectively. All of them in a spectral region (0.9-1.05) $\lambda_{0} / \lambda$ hit in limits of a modification $N_{p}$ at an appropriate angle $\varphi$. In a visible region of a spectrum at $\lambda_{0}=600 \mathrm{~nm}$ the region $(0.9$ 1.05) $\lambda_{0} / \lambda$ corresponds to a modification $\lambda$ from 666 up to $570 \mathrm{~nm}$. Therefore, IP has not only wide aperture, but also broadband.

\section{Summary}

The two component periodic multilayer coatings are a success for solving of the different design problems. Using the symmetric periods with unequal thickness individual layers is essentially simplified the design procedure and have a large potential possibilities. And the modern equipment permit to realize the most complicated coatings.

\section{References}

1. H.A. McLeod, Thin-Film Optical Filters, Adam Hilger, London (1985).

2. A.P.Ovcharenko, E.A. Lupashko, Unequal thicknesses multilayer dielectric coatings, Opt. and Spectr. 55(3), pp. 537-541.

3. I. Fekeshgazi, Yu. Pervak, Broad-band two component unequal layer thicknesses periodic multilayer dielectric systems, SPIE Proceedings, 1983, pp. 280-281 (1993).

4. Yu.A. Pervak, I.V. Fekeshgazi, Anti-reflecting coatings on base of symmetrical periods, Ukr.Journ.Phys., 39(8), pp. 934937 (1994).

5. Yu.A. Pervak, I.V. Fekeshgazi, Optical properties of unequal thinckness multilayer coatings with symmetrical periods at sloping incidence, Ukr.Journ.Phys., 39 (6), pp. 668672 (1994).

6. Yu.A. Pervak, I.V. Fekeshgazi, Spectral properties of the interference polarizers, J.Appl.Spectr. 61 (5-6), pp. 485-490 (1994)

7. Yu.A. Pervak, I.V. Fekeshgazi, Spectral properties of the prism interference polarizers, Opt. and Spectr. 77 (5), pp. 843-846 (1994).

8. Yu.A. Pervak, I.V. Fekeshgazi, Optimization of the Design of Prism Interference Polarization Splitters, Opt. and Spectr. 86 (1), pp. 138-142 (1999).

9. P.B. Clapham, M.J. Downs, and R.J. King, Some Applications of Thin Films to Polarization Devices, Appl. Opt., 8 (10), pp. 1965-1974 (1969).

10. R.S. Sokolova, and T.N. Krylova, The interference polarizers for UV region of spectrum, Opt. and Spektr., 14 (3), pp. 402405 (1963).

11. T.N. Krylova, and R.S. Sokolova, The conditions for receiving line polarization light by help of interference polarizers, Opt.-Mekh. Prom-st., (2), pp. 6-9 (1965).

12. Yu.N. Konoplev, Yu.A. Mamaev, A.Yu. Safronov, et al., Thin-Film interference polarizer for spectral region 745-800 nm, Opt. and Spektr., 73 (4), pp. 823-827 (1992).

13. L.A. Zhdanova, and G.D. Pridatko, The investigation of the optical properties of the interference polarizers at spectral region 0.25-1.2 mkm, Opt.-Mekh. Prom-st., (6), pp.7-10 (1984).

14. L.A. Zhdanova, Polarization elements and devices on base of interference dielectric coatings, Opt. and Spektr., 71 (5), pp.856-860 (1991).

15. M.J. Banning, Practical methods of making and using multilayer filters, Opt. Soc. Am., 37, pp. $792-797$ (1947).

16. J.A. Dobrowolski, and A. Waldorf, High-performance thin film polarizer for the UV and visible spectral regions, Appl. Opt., 20 (1), pp. 111-116 (1981).

17. M. Gilo, and K. Rabinovitch, Design parameters of thin-film cubic-type polarizers for high power laser, Appl. Opt., 26 (13), pp. 2518-2521 (1987).

18. V.G. Borodin, V.M. Miggel, S.L. Potapov, etal., High aperture interference polarizers for laser devices, Opt.-Mekh. Prom-st., (9), pp. 49-52 (1990).

19. J.A. Dobrowolski, and A. Waldorf, Liquid polarizing filter operates over wide range, Laser Focus, 17 (9), pp.45-46 (1981).

20. P.P. Yakovlev, and B.B. Meshkov, Interference analog of the Ahenvald polarizer, Vopr. Radioelektron., Ser. Obshch. Vopr. Radioelektron., (7), pp. 126-129 (1985). 